\title{
Graves' Ophthalmopathy Misdiagnosed as Relapsing Conjunctivitis
}

\author{
Irini P. Chatzirallia Evgenia Kanonidou ${ }^{a}$ \\ Petros Keryttopoulos $^{b}$ Dionyssia Papadopoulou ${ }^{c}$ \\ Leonidas Papazisis ${ }^{a}$ \\ Departments of a Ophthalmology, ${ }^{b}$ Internal Medicine, and 'Surgery, General \\ Hospital of Veroia, Veroia, Greece
}

\section{Key Words}

Conjunctivitis · Graves' ophthalmopathy · Hyperthyroidism

\begin{abstract}
A 59-year-old female patient presented at the outpatients' Department of Ophthalmology with epiphora, eyelid swelling, and a foreign body feeling in the right eye. The symptoms were present for 4 months, and the patient was treated as suffering from relapsing conjunctivitis. The slit lamp examination revealed keratitis due to exposure, related with the deficient closure of the eyelids. There was a $2 \mathrm{~mm}$ difference in the readings with the Hertel exophthalmometry examination between the eyes. Her medical history was clear, and she was referred for computed tomography of the orbits and brain and biochemical examinations $\left(\mathrm{FT}_{3}, \mathrm{FT}_{4}\right.$, and $\left.\mathrm{TSH}\right)$ to investigate the presence of an intraorbital mass. $\mathrm{FT}_{3}$ was significantly increased and $\mathrm{TSH}$ was accordingly low, indicating the diagnosis of Graves' disease, which presented without other signs and symptoms apart from ophthalmopathy. Computed tomography scan excluded the diagnosis of an intraorbital mass. Therefore, it is important not to underestimate the ocular manifestations of systemic diseases.
\end{abstract}

\section{Introduction}

Graves' disease usually affects females, and in $25-50 \%$ of the cases coexists with ophthalmopathy [1]. Thyroid eye disease is a relatively rare condition, which affects 2.916.0 of cases per 100,000 per year [2]. Here, we describe an interesting case of Graves' ophthalmopathy, which was misdiagnosed and treated as relapsing conjunctivitis. 


\section{Case Report}

A 59-year-old female patient presented at the outpatients' Department of Ophthalmology with the symptom complex of conjunctival hyperemia, epiphora, irritation, and a foreign body sensation in the right eye (fig. 1). She had been examined by multiple ophthalmologists prior to our evaluation, as the symptoms were present for 4 months, and she was treated as suffering from relapsing conjunctivitis.

A full ophthalmic evaluation was performed, and the slit lamp examination revealed keratitis due to exposure, which was related to the widening of the interpalpebral fissure and accelerated tear film evaporation in the right eye. The other eye was normal, as well as the intraocular pressure bilaterally. The patient had no poor aqueous production, and the Schirmer test was normal. There was also a $2 \mathrm{~mm}$ difference in the readings with the Hertel exophthalmometry examination between the eyes, while a sublte deficiency in the supralateral gaze position was revealed in the eye movements examination. In addition to this, a detailed medical history was carried out, with a clear result. The patient also underwent thorough clinical examination, and it is worthy to mention that she had neither diabetes nor other systemic diseases.

The patient was referred for computed tomography (CT) of the orbits and brain and biochemical examinations $\left(\mathrm{FT}_{3}, \mathrm{FT}_{4}\right.$, and TSH) to exclude the presence of an intraorbital mass and investigate the cause of exophthalmos. $\mathrm{FT}_{3}$ was significantly increased $(4.9 \mathrm{ng} / \mathrm{dl}$, normal ranges: $0.9-1.8 \mathrm{ng} / \mathrm{dl})$ and TSH was accordingly low (0.03 mIU, normal ranges: $0.17-4.65 \mathrm{mIU})$, indicating the diagnosis of Graves' disease, although there was no goiter. CT scan revealed enlargement of the extraocular muscles and excluded the diagnosis of an intraorbital mass (fig. 2). The other laboratory findings were all normal. Three months later, the ophthalmopathy improved on antithyroid treatment [Thyrostat $5 \mathrm{mg}$ (Carbimazole), 3 tablets 3 times/day]. Moreover, artificial tears (Systane Ultra ${ }^{\circledR}$ lubricant eye drops, Alcon; 1 drop 6 times/day) caused reduction of symptoms, while resolving corneal staining and hyperemia. Therefore, the patient suffered from undiagnosed Graves' disease, without other signs and symptoms apart from ophthalmopathy.

Written informed consent was obtained from the patient.

\section{Discussion}

The classic symptoms of the thyroid eye disease include eyelid retraction, exophthalmos, dysmotility, and diplopia [1-5]. Nevertheless, there are patients who present only ocular surface irritation, hyperemia and conjunctivitis [2, 3]. Although these latter findings are well established, ocular manifestations are frequently overlooked, and diagnosis of thyroid disease is usually made when the classic features manifest. In addition to this, thyroid eye disease is often misdiagnosed as allergic or relapsing conjunctivitis, as tearing and hyperemia are the predominant features $[2,5]$. In such cases, the presence of eyelid retraction and restricted eye movements helps to differentiate thyroid eye disease from other causes of periorbital edema [2, 3, 5]. Moreover, thyroid function tests $\left(\mathrm{FT}_{3}, \mathrm{FT}_{4}\right.$, and $\left.\mathrm{TSH}\right)$ can be helpful in the diagnosis of thyroid ophthalmopathy and should be included in the routine work-up.

In conclusion, it is important not to underestimate the ocular manifestations when examining a patient, as potentially serious and systematic conditions may underlie these symptoms. Furthermore, early recognition of Graves' orbitopathy is important, as response to medical treatment is dependent on the duration of the disease.

\section{Conflict of Interest}

None. 


\begin{tabular}{l|l|l|l} 
Case Reports in & $\begin{array}{l}\text { Case Rep Ophthalmol 2010;1:53-55 } \\
\text { DOI: 10.1159/000320582 }\end{array}$ & $\begin{array}{l}\text { Published online: } \\
\text { September 13, 2010 }\end{array}$ & $\begin{array}{l}\odot \text { 2010 S. Karger AG, Basel } \\
\text { ISSN 1663-2699 } \\
\text { www.karger.com/cop }\end{array}$ \\
\hline
\end{tabular}

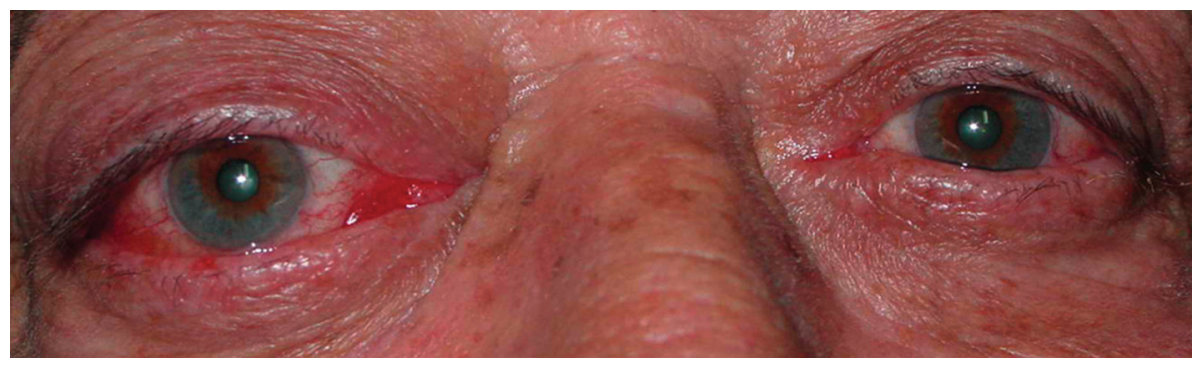

Fig. 1. Clinical image of the patient showing the conjunctival hyperemia and exophthalmos.

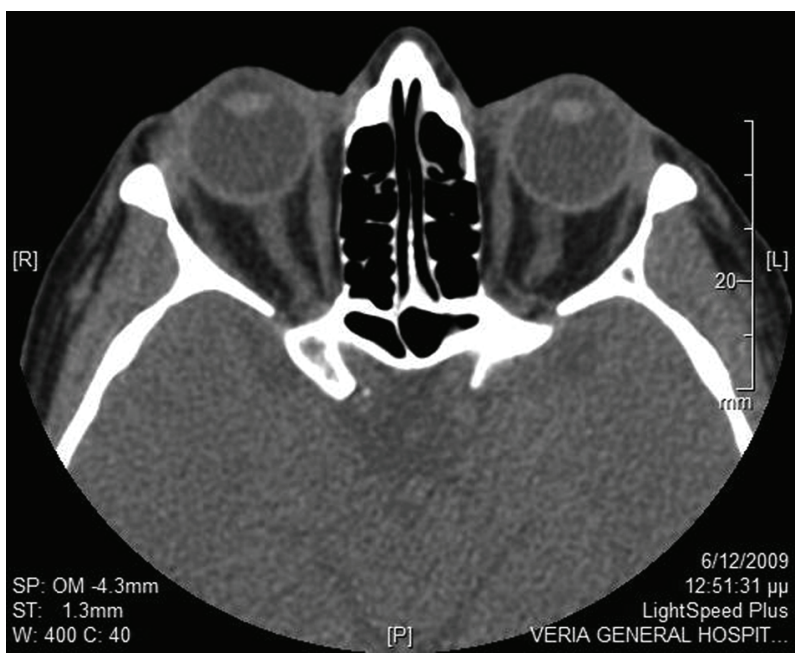

Fig. 2. CT excluded an intraorbital mass and revealed enlargement of extraocular muscles.

\section{References}

1 Kuriyan AE, Phipps RP, Feldon SE: The eye and thyroid disease. Curr Opin Ophthalmol 2008;19:499-506.

2 Perros P, Neoh C, Dickinson J: Thyroid eye disease. BMJ 2009;338:b560. DOI: 10.1136/bmj.b560.

- 3 Gupta A, Sadeghi PB, Akpek EK: Occult thyroid eye disease in patients presenting with dry eye symptoms. Am J Ophthalmol 2009;147:919-923.

-4 Costa RM, Dumitrascu OM, Gordon LK: Orbital myositis: diagnosis and management. Curr Allergy Asthma Rep 2009;9:316-323.

5 Bartley GB, Fatourechi V, Kadrmas EF, Jacobsen SJ, Ilstrup DM, Garrity JA, et al: Clinical features of Graves' ophthalmopathy in an incidence cohort. Am J Ophthalmol 1996;121:284-290. 